\title{
Surfaces
}

\section{Musique et postmodernité}

\section{Jean-François Lyotard}

Volume 6, 1996

LES ÉCONOMIES DISCURSIVES DU SAVOIR ET DE LA CULTURE DANS

LE SILLAGE DE L'OEUVRE DE BILL READINGS

THE DISCURSIVE ECONOMIES OF KNOWLEDGE AND CULTURE,

WITH CONSTANT REFERENCE TO THE WORK OF BILL READINGS

URI : https://id.erudit.org/iderudit/1064848ar

DOI : https://doi.org/10.7202/1064848ar

Aller au sommaire du numéro

Éditeur(s)

Les Presses de l’Université de Montréal

ISSN

1188-2492 (imprimé)

1200-5320 (numérique)

Découvrir la revue

Citer cet article

Lyotard, J.-F. (1996). Musique et postmodernité. Surfaces, 6.

https://doi.org/10.7202/1064848ar d'utilisation que vous pouvez consulter en ligne. 


\section{Musique et postmodernité}

Jean-François Lyotard

Surfaces Vol. VI. 203 (v.1.0F - 27/11/1996) - ISSN:

1188-2492

Tout texte reste la propriété de son auteur. Néanmoins, Surfaces demande d'être citée à l'occasion de toute autre publication du texte en question.

Quand il n'y a plus de règles du tout, vient à son heure celle de l'atonalisme. De ce qui précède, il ne reste rien de rien. Il reste pourtant le son [...]. Or le son prodigue d'immenses restes .

Je voudrais d'abord partir de cette observation à la fois mélancolique et joyeuse écrite par Pierre Schaeffer il y a dix ans pour situer, encore une fois, ce qu'il en est de la question postmoderne.

Dans La Condition postmoderne, qui est un Rapport écrit il y a quelques douze ans pour une institution québécoise, j'essayais d'entendre et de faire entendre un événement. Prêter l'oreille à un événement est la chose la plus difficile du monde. Un événement n'est pas ce qui occupe la première page des journaux. Il est quelque chose qui survient, qui vient en sortant de rien. Comme tel, ce quelque chose n'est encore rien: on ne saurait le qualifier ni même le nommer. On n'y est pas préparé, on n'a pas de quoi l'accueillir ou le placer dans un système de signification, de quoi l'identifier. Et pourtant s'il advient, c'est qu'il touche à quelque surface où il inscrit sa trace: une conscience, un inconscient, individuels ou collectifs. Peut-être même a-t-il à inventer cette surface pour s'inscrire. 
Cette trace n'est pas compréhensible aussitôt. C'est une trace étrange, une trace d'étrangeté, en attente de son identification, alors que l'événement est déjà passé. C'est pourquoi il est difficile de prêter l'oreille à l'événement: il n'est déjà plus là quand ce qu'il est n'est pas encore là.

Donc le Rapport essayait de dire de quel événement les sociétés développées (c'était l'objet spécifié par la commande) étaient touchées à la fin des années 70. Je pensais cet événement en Occidental. L'Occident est cette région du monde humain qui invente l'Idée de l'émancipation, de l'auto-constitution des communautés par elles-mêmes, et qui essaie de réaliser cette Idée. La mise en actes se soutient du principe que l'histoire est l'inscription du progrès de la liberté dans l'espace et le temps humains. La première expression de ce principe est chrétienne, la dernière marxiste. La Condition postmoderne désignait brièvement le discours de ces philosophies de l'histoire du nom de grand récit.

Il y a plusieurs grands récits co-existant dans la pensée occidentale. La condition postmoderne résulte de ce que les grands récits cessent d'être crédibles. Ils ne peuvent plus légitimer au nom du progrès les bienfaits et les méfaits que l'Occident a répandus sur lui-même et sur le monde au cours des siècles. Je n'expliquerai pas les motifs que l'on peut trouver pour justifier ce diagnostic. Ils sont évidemment discutables, et le diagnostic luimême est trop massif pour ne pas être suspect. Pourtant la récente implosion des États qui se réclamaient du grand récit marxiste peut apporter une sorte de vraisemblance à l'hypothèse faite dix ans auparavant.

On objectera que la disparition de l'Empire soviétique prouve plutôt la vitalité du grand récit capitaliste. Je répondrai qu'il n'y a pas de grand récit capitaliste. Le capitalisme développe tout chose, mais le développement n'est pas nécessairement le progrès de la liberté. Le développement complexifie les relations entre les éléments d'un système quel qu'il soit de telle sorte que le système s'en trouve plus performant. Le plus souvent aux dépens d'un autre, qui sera condamné à disparaître s'il n'améliore pas ses performances. C'est un processus objectivement réglé selon une loi de différenciation croissante (entropie négative). Il opère sans finalité. Il est indifférent au bien et au mal, ou, si l'on préfère, le seul bien qu'il connaisse est l'amélioration des performances. On ne peut l'influencer, l'orienter, le modérer qu'en travaillant dans son sens. Cela est évident dans les affaires économiques ou dans la recherche techno-scientifique. 
La condition postmoderne est celle des humains quand ils sont saisis dans ce processus qui à la fois développe leurs pouvoirs et exige leur asservissement. Elle est notamment marquée par l'introduction massive d'automates capables d'effectuer des opérations de type mental (calculs, combinaisons, élaboration de problèmes, diagnostics) et de les faire exécuter par des automates physiques. Le monde dit développé ou en développement ne se soutient d'aucun récit qui légitimerait son cours par une émancipation. Les systèmes deviennent simplement plus puissants.

\section{2.}

Tournons nous à présent vers l'histoire de la musique. La musique occidentale a-t-elle été affectée par un événement analogue? La question est naïve, nous verrons pourquoi. Mais à la prendre comme elle est posée , on peut essayer d'en élaborer un aspect.

Dans Philosophie de la nouvelle musique, Adorno suggère que les technologies nouvelles permettent au matériau sonore de se libérer des contraintes dans lesquelles la tradition musicale de l'Occident le tenait subordonné. Et qu'ainsi la maîtrise possible du matériau s'accroît parce que d'autres règles deviennent concevables. La question de ce qu'on appelle le matériau, qui est celle de la matière, est en effet une question décisive pour la musique contemporaine, comme pour tous les arts. Encore faut-il convenir de ce qu'on entend par matériau ou matière.

L'histoire de la musique occidentale peut être pensée globalement comme le grand récit de l'émancipation du son. On dirait que les compositeurs cherchent à retrouver ce que peut le matériau sonore à travers les règles et les coutumes de la composition dont ils héritent. A travers, c'est-à-dire avec elles et contre elles: contraintes sur les timbres imposées par l'instrumentation, sur les durées et les rythmes réglés par la mesure, sur les hauteurs déterminées par les échelles et les modes, sur l'assemblage des unités sonores soumis aux principes de l'harmonie et de la mélodie.

L'analyse critique de ces règles les fait apparaître comme contingentes. Elle délivre à son terme l'élément 
matériel comme le seul objet musical, la vibration de l'air avec ses composantes, fréquence, durée, amplitude, la couleur, l'attaque, que l'acoustique physique et psychophysiologique analyse. De nouvelles manières de traiter le son s'avèrent possibles et permises, bien différentes de ce que la tradition permettait. Et les recherches techno-scientifiques viennent ainsi confluer avec le travail d'émancipation du son entrepris, de leur côté, par les artistes.

Le compositeur d'aujourd'hui a le sentiment que tout est possible et qu'il doit inventer pour chaque oeuvre non pas seulement sa forme musicale, mais les règles de la musique. Comme dans les savoirs économiques ou sociopolitiques, un certain cynisme peut se faire jour dans la pratique artistique contemporaine. Le postmodernisme revêt souvent ce sens: tout peut être intéressant, la citation, l'ornementation, le kitsch, la parodie, le néoceci et le post-cela. A condition toutefois que le travail trouve une bonne réception et que l'oeuvre soit un succès culturel . La règle du marché des objets culturels vient alors pallier l'absence des règles artistiques, subordonnant la production des oeuvres aux critères de leur communication et de leur circulation, - ce qui est la définition du travail réduit au statut d'une marchandise.

\section{3.}

Depuis la publication de la Condition postmoderne, il s'est avéré nécessaire de corriger plusieurs implications que les lecteurs et les critiques se sont jugés autorisés à en tirer. Le cynisme que je viens de noter n'est de fait qu'une réaction puérile au malaise, à la mauvaise conscience, à l'angoisse qui envahit le monde développé du fait qu'il ne sait pas comment justifier ce qui lui arrive. Il est également superficiel d'interpréter le postmoderne comme une révolution du moderne. L'idée même de révolution appartient à la représentation moderne d'un progrès subit accompli dans la marche vers la liberté. Elle n'a pas de sens en dehors d'une conception qui donne à l'histoire l'émancipation des hommes comme fin. Si le terme postmoderne a quelque sens, il doit se soustraire à cette philosophie de l'histoire, puisqu'il est censé marquer la ruine des grands récits. 
Mais surtout, une fois débarrasser de cette périodisation désuète on découvre, en se tournant vers les oeuvres de l'art surtout, que leur modernité ne signale rien d'intrinsèque en elles. C'est l'accueil que les contemporains leur font qui les désignent comme telles. Mais ce qui fait la valeur artistique d'une oeuvre est tout à fait indépendant de leur réception. Cette valeur n'est due qu'à un geste singulier, à un paradoxe de temps, d'espace et de matière dont l'oeuvre est l'enregistrement sensible. Peu importe si ce geste est ou n'est pas compatible avec le goût, avec les Écoles, avec le style contemporain. En vérité, du reste, la manière artistique de ce qu' on appelle une époque n'est jamais homogène. Entre Pergolèse et Gesualdo, entre le Beethoven des derniers Quatuors et Tchaïkovsky des concertos, qui peut dire ce qui est proprement renaissant ou romantique? Et quand Varèse introduit des enregistrements de chants d'oiseaux ou de sirènes d'usine dans ses pièces musicales, est-il moderne ou postmoderne?

C'est une erreur, grave et courante, d'imposer aux oeuvres de l'art une classification par périodes ou par écoles. On ne classe en réalité que des produits culturels, qui appartiennent en effet aux phénomènes observables de la réalité historique, comme le sont les événements politiques, les mutations démographiques, les changements économiques. Mais ce qu'il y a d'art dans les oeuvres est indépendant de ces contextes, même s'il ne se manifeste qu'en eux et à leur occasion. L'art de l'oeuvre est toujours un geste d'espace-tempsmatière, l'art de la pièce musicale un geste d'espacetemps-son.

Ce geste n'est pas le fait de l'auteur. Le travail de l'auteur est de laisser le son faire un geste qui paraît excéder l'audible et d'en consigner la trace dans l'espace-temps-son qui détermine le champ de l'audible. Ce geste n'est pas moins puissant en émotion dans une musique nô que dans un lied de Schumann. La lumière d'une fresque de Piero della Francesca n'est pas moins invisible que celle d'une aquarelle de Cézanne. Les gestes, qui ne sont pas les contenus ni les formes mais le pouvoir absolument émouvant de l'oeuvre, ne font pas de progrès au cours de l'histoire. Il n'y a pas d'histoire de l'art comme geste mais seulement comme produit culturel. Le pouvoir d'affecter la sensibilité au-delà de ce qu'elle peut sentir n'appartient pas au temps chronologique. C'est seulement ce qu'on appelle la fonction de l'art, la trace du geste recueillie dans les 
communautés humaines, qui se transforme avec cellesci, et qui peut être périodisée.

\section{4.}

Les musiques occidentales se sont intéressées depuis longtemps au problème posé par le timbre. La tradition européenne découpe le continuum sonore selon un ensemble de variables discontinues strictement maîtrisées. Dans ses séminaires sur le timbre, Pierre Boulez insiste sur la résistance que la nature d'un son comme celui que produit la simple percussion d'un gong peut opposer à ce découpage. Il est en effet difficile de l'analyser selon les composantes recensées par la théorie harmonique et de l'inclure dans une ligne mélodique dite pure . Ce son révèle une singularité et une complexité presque sauvages. On peut obtenir des sons de cette nature au moyen d'un synthétiseur. Celui-ci peut en effet produire toutes les nuances intermédiaires entre deux degrés fixés sur l'échelle chromatique. Cette extension de l'audible a été inaugurée de façon systématique dans la musique par le sérialisme de Vienne.

Cependant Debussy a déjà recours à des gammes orientales. Éric Satie et bien d'autres s'attaquent au principe de la résolution. Les percussions sur lesquelles Boulez appuie sa méditation concernant le timbre, proviennent des musiques indiennes, chinoises et japonaises. John Cage de son côté se réfère explicitement à ces dernières pour légitimer une musique qui cherche à déjouer les règles convenues de la composition. Il ne s'agit en aucun cas d'une idéologie orientaliste. Il s'agit d'émanciper le son de la fonction narrative que lui assignent la plupart des formes occidentales modernes, la sonate, la symphonie, le poème symphonique. Il s'agit de faire entendre des timbres et des clusters de timbres en neutralisant l'expressionnisme lyrique, pathétique ou héroïque auquel l'Occident les a généralement soumis.

Dans cette stratégie d'émancipation de la matière sonore, il serait vain d'accorder le prix de postmodernisme ou de modernisme à telle ou telle école. La procédure aléatoire observée par les compositeurs de la tendance Cage penche vers une sorte de minimalisme sonore. La technique savante employée par Boulez paraît au contraire tournée vers la multiplication des 
contraintes imposées aux paramètres du son. Elle recueille ce qu'il y a de plus intrépide dans les recherches de Debussy, Schoenberg et Webern, Bartok Varèse, les percussions de jazz. Ces expérimentations sont pour Boulez autant d'approches de gestes sonores que l'oeuvre musicale tente de faire résonner pour les oreilles humaines. Mais pour une tendance comme pour l'autre, il s'agit non pas de déconcerter l'auditoire, mais, comme l'écrivait Varèse, de dépasser les limites de l'audibilité . Nos oreilles sont sourdes à ce que le son peut. Il faut rendre à l'écoute la puissance de se prêter à l'inaudible.

Ce paradoxe des musiques contemporaines est celui de tout art en tout temps. Tout art s'adresse à la sensibilité, c'est-à-dire au corps en tant qu'il peut être affecté par des sensations, sonores, visuelles, etc... Il doit, par hypothèse, offrir un arrangement d'éléments sensibles par lequel le corps est touché. L'énigme de cette touche est qu'elle affecte la pensée en même temps que le corps. La séparation du corps et de la pensée n'a aucune réalité pour l'expérience artistique. L'art suppose toujours une sorte d'incarnation, une pensée dans le corps, un corps qui soit immédiatement une pensée affectée. Cette supposition n'est un mystère ou un scandale que pour une représentation dualiste de l'âme et du corps. Une oeuvre est toujours une chose sensible au corps. Le corps n'en est pas seulement informé, comme un machine l'est d'un objet, il en est affecté. Il découvre son affectivité à l'occasion de l'oeuvre.

Le paradoxe propre à l'art consiste à donner à sentir à cette pensée-corps un arrangement sensible et émouvant, certes, mais qui suggère aussi la présence en lui d'un geste qui excède la capacité de cette penséecorps. En disant arrangement, on reconnaît qu'il s'agit toujours de formes, et en ce sens, aussi désoeuvrée que soit sa forme, l'oeuvre reste une oeuvre. Mais par excès, on désigne un secret immanent à la forme qui la transcende. Il y a dans l'oeuvre un reste qui défie la réception ou la perception ordinaire et qui défiera le commentaire. Ce secret n'appelle aucune mystique. Un secret n'est pas un mystère. Au delà ou en deçà de la sensibilité ordinaire, il doit pourtant lui faire signe. L'oeuvre peinte doit être visible, la pièce musicale audible. Simplement le visible laisse entrevoir un 
invisible du visuel, l'audible sous-entend un inaudible du sonore.

C'est pourquoi j'ai parlé d'espace-temps-matière. L'inaudible et l'invisible n'appartiennent pas à un substrat suprasensible qui échapperait entièrement à la condition ordinaire de l'espace-temps-matière. En ce cas, seule la philosophie métaphysique ou critique pourrait parler de lui. Mais l'inaudible est un geste dans l'espacetemps-matière du son, et il y fait signe d'une présence qui n'est pas présentable.

Il faut le répéter: le geste n'est pas fait par le compositeur, il n'exprime aucune subjectivité. Le délice ou le désordre qu'il procure à la subjectivité du compositeur ou de l'auditeur suppose au contraire une sorte de suspension ou de décomposition de cette subjectivité. Car la présence du geste dans la présentation des formes entraîne le dénouement des synthèses sur lesquelles la subjectivité est construite. Son temps, son espace, la matérialité des sensations qui l'affectent sont suspendus. La présence n'est pas ellemême sentie puisqu'elle ne satisfait pas aux conditions de lieu, de moment et de sensorium qui sont celles de la sensibilité subjective.

Ce geste ne doit donc nullement être confondu avec l'expression. Le romantisme nommait Stimmung une disposition de la pensée, une destination de l'âme dont l'art serait l'expression. Et la musique était considérée comme l'art le plus propre à exprimer ce qui, dans le sujet, transcende sa connaissance de soi, c'est-à-dire sa mystérieuse Stimmung. En penseur moderne ou postmoderne, Adorno rompt d'un trait avec le romantisme: l'expression, écrit-il, est une contre-façon .

Pourquoi cela? Parce que l'art n'a pas pour destination d'exprimer le mystère de l'incarnation. Au contraire, l'art suppose nécessairement une pensée-corps, la capacité d'être affecté par le sensible. L'art n'a rien à exprimer d'elle, il a à l'excéder.

La Stimmung a besoin de la forme narrative pour s'exprimer, en quelque art que ce soit. Le poème musical, la symphonie, la sonate, le lied racontent toujours l'odyssée, heureuse ou malheureuse, d'une subjectivité: épopée, tragédie, élégie. Même le leitmotiv de l'opéra wagnérien, les dissonances dans les lieder de Malher ou les poèmes de Stravinsky, narrent le drame d'une subjectivité désaccordée d'avec elle-même.

Mais la transcendance du geste musical ne consiste pas dans le mystère d'une pensée pure ayant à subir la 
passion d'une vie et d'une mort sensibles, qu'elles soient ou non rédemptrices. S'il fallait situer l'enjeu (mais qu'est-ce qu'un enjeu ici?) de la lutte de la musique pour l'espace-temps-son, on le trouverait non pas tant du côté du sujet, mais de la langue. Le conflit entre Boulez et Cage ne porte certainement pas sur l'expression de la subjectivité, mais sur le rôle de la langue comme matériau articulé. Cage pense qu'on peut se soustraire à l'articulation (et à la composition, qui en est la forme suprême) en recourant au silence, à la contingence, à l'événement, à la rencontre imprévisible d'une pièce pour piano et d'un bruit de métro dans la rue. Cage soutient que le bruit est un son musical brut, non tempéré. Il est en cela fidèle à la pensée de Varèse.

De son côté, Boulez lui aussi procède de cette pensée. Mais il en tire la conclusion que l'on doit sur-articuler toutes les composantes du langage musical pour lui arracher sa matière-son inaudible. Car à simplement laisser être les sons comme ils arrivent, selon la formule de Cage, on n'entendra jamais que ce qui permet le filtrage du continuum sonore par notre organisation physiologique, psychique et culturelle. Or il s'agit pour la musique d'esquisser une autre nature acoustique et sonore. Boulez n'a pas confiance dans la spontanéité de notre nature musicale, qui par elle-même n'est que ce qu'elle est. Cage pense que l'autre nature est là, potentielle dans la nature musicale, comme une puissance de paradoxes sonores, qu'il n'est pas opportun ni nécessaire de violenter. Je ne trancherai pas entre ces deux philosophies de la matière sonore. Il est certain qu'elles ont un projet commun: affranchir cette matière de son enveloppe formelle conventionnelle.

\section{6.}

Rappelons-nous la phrase écrite par Adorno dans Philosophie de la nouvelle musique: Avec la libération du matériau, la possibilité de le maîtriser s'est accrue . Le matériau est-il ce qui se nomme ici matière? Je ne pense pas. La notion de matériau qui vient d'Aristote par la tradition des métiers et des savoir-faire, implique toujours le concept d'un usage. Le matériau est cette matière à laquelle la main et la pensée du fabricant vient donner une forme. Le matériau est conçu essentiellement comme une matière en attente, en souffrance, d'une forme qui l'achève et lui donne acte, 
comme une puissance qui n'est pas encore actualisée ou actée.

Or pour autant que les arts, mais aussi les sciences, sont guidés par le projet, absolument aporétique, de faire sentir ou de penser la matière, ils supposent toujours celle-ci autre que sensible ou pensable. Ils supposent qu'elle n'a même pas en elle-même l'attente de sa forme, qu'elle n'obéit à aucune finalité. Elle ne $s^{\prime}$ adresse pas à la pensée, elle ne s'adresse pas non plus à la penséecorps, à la sensibilité. Pour s'adresser, il faudrait qu'ellemême parle déjà une sorte de langage.

L'adresse et la destination supposent une relation, que nos contemporains appellent pragmatique, celle d'un je, d'un tu et d'un il ou elle, et cette relation est donnée seulement dans le langage articulé humain, par ses fonctions pronominales. On objectera que la couleur d'un fuchsia qui attire ou appelle les abeilles et les guêpes à le butiner est une sorte de message (ou de phrase) qui est destiné à ces animaux, et que c'est une preuve que la destination ne requiert pas le langage articulé.

On peut le concéder. Mais on n'oubliera pas que la corrélation de l'abeille et du fuchsia peut aussi s'expliquer comme un montage bio-physique, au titre d'un réflexe, d'un tropisme ou d'un instinct . Ce qui est certain, quelle que soit l'explication, est que cette corrélation paraît invariable, sauf si l'on introduit un conditionnement supplémentaire. Alors que le pouvoir de s'adresser qui est impliqué dans une phrase de langage articulé n'est pas déjà bloqué sur un genre spécifique de destinataire comme la couleur du fuchsia l'est pour l'abeille ou comme la convoitise laborieuse de celle-ci l'est pour cette couleur, selon une complémentarité préétablie qui n'est presque pas négociable. La destination inscrite dans la phrase articulée est ouverte à un destinataire certes, mais inconnu. Cette destination suppose tout simplement qu'il y a un autre et qu'il peut, lui aussi, parler. Toi est potentiellement je. Même ce qui fut lui ou elle, celui ou celle dont il a été parlé, peut, le cas échéant, être adressé et s'adresser à son tour.

En essayant de penser la matière-son comme inadressée, on touche à l'aporie des musiques contemporaines et à la vérité profonde qu'elles révèlent dans l'art selon cette aporie. D'abord, comme tous les arts, elles ignorent la division que je viens de rappeler entre langage du corps et langage articulé. Il y a aussi quelques prémontages sonores du corps humain, de nature et de culture, et la musique a toujours été l'effort de les excéder. Mais 
ensuite, et surtout, ce dépassement lui-même doit être dépassé.

Car il enferme de nouveau la matière sonore dans le langage de l'harmonie, de la mélodie, de la rhétorique musicales, afin de l'adresser à des auditeurs (des destinataires) qui pourront l'entendre parce qu'ils peuvent en déchiffrer le code, consciemment ou non. En se proposant pour idéal de faire sentir à l'oreille la matière-son - le timbre - affranchie de toute destination, les musiques contemporaines extrémisent leur enjeu jusqu'à l'aporie constitutive de toute musique: faire entendre ce qui se soustrait par soi-même à toute écoute, adresser ce qui n'est pas adressé.

C'est ainsi qu'un peintre me disait un jour: je peins pour les aveugles.

La conjoncture présente des musiques, comme des autres arts, se caractérise à cet égard par une triple convergence: la déconstruction des codes selon lesquels le matériau sonore a été traité dans les traditions populaires et savantes; les recherches en anatomie, physiologie, psychologie et médecine portant sur les capacités du corps humain (pris comme objet) à supporter des situations auditives étrangères à la nature et à la culture; la prospection de ces situations au moyen des technologies informatiques et électroniques.

Tout se passe aujourd'hui comme s'il s'agissait pour le musicien de faire entendre comment le timbre s'entend lui-même. A l'école des aquarellistes japonais, Cézanne aussi cherchait à faire voir la couleur comme elle se voit elle-même. Un musicien français, Jean-Claude Eloy, souhaite une musique qui ne serait pas faite de notes dans un rapport d'intervalle, mais des clusters dotés d'une vie interne. Je souligne vie interne, qui dit l'aporie dont je parle. Et ce n'est pas par hasard si le même compositeur conclut son voeu par un appel au laconisme de ce qu'il nomme le son japonais : un peu d'eau dans un jardin .

Si la matière sonore n'attend rien de sa mise en forme, il faut dire aussi qu'elle n'attend pas d'être entendue pour vibrer ou sonner. Cet idéal aporétique de la musique se dit en allemand Tonkunst, un savoir du timbre. Cette matière qui se soustrait à la destination n'est pas le matériau, lequel est seulement le support d'un message. Elle est immatérielle. Les physiciens savent que ce que nous appelons matière est de l'énergie formée en corpuscules et en objets. L'oeuvre d'art porte témoignage que les objets n'existent pas, qu'ils sont des 
traces filtrées, encodées et décodées par notre sensibilité corporelle et nos langues, les traces d'un pouvoir qui les excède.

Une dernière observation, aussi brève que possible. Il est devenu évident, à l'échelle planétaire, qu'il n'y a pas une école, un style, une technique qui garantiraient un accès privilégié au geste d'espace-temps-matière qui transcende la sensibilité auditive. Il résulte de cette conviction un effet d'éclectisme, même de babelisme, dont on croit parfois pouvoir tirer une leçon de scepticisme, cynique ou désespéré. L'histoire de Babel, dans la Genèse, raconte que les peuples, avant d'entreprendre la construction de la Tour parlaient des langues différentes et qu'ils se comprenaient. Le Seigneur interrompit l'édification du monument unitaire en rendant les langues incompréhensibles les unes aux autres. Il fallut inventer la traduction.

Certains aujourd'hui essaient de reprendre le projet d'un monument unique, un concert des nations, de leurs langues et de leurs musiques, une transparence. Ce monisme reste toujours dangereux. C'est aujourd'hui celui de l'objet culturel, qui vient occuper la place singulière des oeuvres. Nous sommes dans la situation de l'époque de Babel. Les musiques sont différentes, mais elles s'entendent les unes les autres. Elles ne s'échangent pas, elles se rencontrent. Si l'on prétend homogénéiser leur diversité, on obtient une surdité forcée. Il faut que la tour ne soit pas construite. Il y a sans doute des musiques, comme des langues, qui remplissent la fonction de véhicule sonore, rythmique notamment, généralisé. Mais elles sont de l'ordre de la monnaie, un équivalent général. Elles assurent une identité reconnue dans le concert des nations. Leur sens ou leur fonction existe en dehors de leur matière sonore.

Mais il est du destin de chaque musique, affrontée à l'aporie matérielle de l'art, d'approfondir sa singularité. Elle y est poussée notamment, je l'ai dit pour les musiques occidentales, par sa rencontre avec les autres. Il ne s'agit pas d'emprunter à celles-ci quelque motif ou quelque technique. Il s'agit d'étendre le concept de l'inaudible et les moyens d'en porter témoignage. Le Seigneur fut sage d'empêcher la Tour.

Accueil Surfaces | Table des matières | Recherche $\underline{\text { Surfaces Home Page | Table of Contents | Search }}$ 